ORIGINAL ARTICLE

\title{
STD trends and patterns of treatment for STD by physicians in private practice in Peru
}

\section{P J Garcia, K K Holmes}

See end of article for authors' affiliations ......................

Correspondence to: King K Holmes, MD, PhD, Center for AIDS and STD, University of Washington, Harborview Medical Center, Box 359931, 325 9th Ave, Seattle, WA 98104, USA: worthy@u.washington.edu

Accepted for publication 19 March 2003

\begin{abstract}
Objectives: To describe trends in STD visits to physicians in private practice in Peru over a 15 year period and in the patterns of treatments used for STD.

Methods: IMS Health conducts for pharmaceutical marketing purposes surveys of a random cluster sample of $1.63 \%$ of practising physicians in Peru, stratified by region and specialty. Physicians record details of diagnoses and treatments for all patients seen during a 7 day period every 6 months. Data collected on selected STD syndromes were retrospectively reviewed over a 15 year period.

Results: The number of first visits for pelvic inflammatory disease (PID) and trichomoniasis, and total visits for genital herpes increased from 1983-5 to 1996-7; while first visits for gonorrhoea and total visits for syphilis have changed little in recent years. Treatment for gonorrhoea usually involved the use of spectinomycin or an aminoglycoside only. Treatments offered for PID were remarkably inadequate and for trichomoniasis often involved products not known to be effective for trichomoniasis or other causes of vaginal discharge.

Conclusions: This form of active surveillance provides information potentially useful to guide policies for prevention and management of STDs and HIV infections in developing countries.
\end{abstract}

$\mathrm{T}$ he global epidemiology of sexually transmitted diseases (STD) has changed rapidly during the AIDS era. ${ }^{1}$ Monitoring epidemiological patterns of STD, and treatments used for STD, helps guide development and evaluations of preventive and therapeutic interventions and health policies. However, existing passive surveillance mechanisms are grossly inadequate. Sporadic local epidemiological studies $^{2}$ and population based surveys $s^{3-6}$ are informative but not generalisable and give no data on longer term patterns of change.

IMS Health conducts active surveillance of clinical diagnoses and of therapies used by physicians in private practice in several countries around the world. IMS Health data, collected primarily for pharmaceutical marketing purposes, have been routinely used for years in the United States by the Centers for Disease Control and Prevention for surveillance of certain non-reportable STDs in the United States, including non-gonococcal urethritis (NGU), pelvic inflammatory disease (PID), genital herpes, genital warts, trichomoniasis, and other vaginal infections. ${ }^{7-9}$ The data have also been used to assess trends in antimicrobial usage for pelvic inflammatory disease (PID). ${ }^{10}$ In the absence of effective surveillance systems for STDs, the IMS Health data provide a potentially useful mechanism for assessing trends in incidence of various STDs or STD syndromes, and for monitoring trends in antimicrobials used for these conditions. This report reviews data collected by IMS over a 15 year period concerning various STDs seen by physicians in private practice in Peru.

\section{METHODS}

In Peru, IMS Health compiles data from diary reports made by a stratified cluster sample of physicians in private medical practice (excluding certain specialties, such as radiology, anaesthesiology, and pathology), stratified by region and by specialties. Every 6 months, physicians sampled record details from all patients seen or contacted in any way during 7 consecutive days, including patients for whom no prescription is written. Each physician reports the following: sex and age, location of visit, payment status, diagnosis, referral, visit status (first or follow up visit), therapy, $\operatorname{drug}(\mathrm{s})$ prescribed, dosage form, drug strength, daily dosage, treatment period, desired effects. Sample data are then projected to national levels. The number of physicians in Peru is estimated to be 23866 , and the sample of physicians participating in each survey has been 390, representing $1.634 \%$ of Peruvian physicians. The lower and upper confidence limits of an NDTI Peru estimate of 200000 physician visits or prescriptions over 2 years (as seen with visits for PID, gonorrhoea, and trichomoniasis in this paper), are 164968 and 235032 respectively (relative error at this level is plus or minus $17.5 \%$ ). This is to say that the true number will fall within the above range $95.5 \%$ of the time. For values lower than 50000 the error is about plus or minus $35 \%$, which is the error that we could expect for the estimates for syphilis in this report). For values lower than 25000 , as with the estimates for genital herpes or chancroid, the error could be as high as plus or minus $50 \%$. We retrospectively reviewed data collected for the years 1983 and 1985, and for 2 year periods from 1990-7 for Peru regarding trends in numbers of visits and treatments recommended for the following major STDs or STD syndromes: syphilis (all stages), gonorrhoea (first visits), genital herpes (all visits), pelvic inflammatory disease (first visits), vaginal trichomoniasis, and chancroid. Diagnoses were coded according to the International Classification of Diseases. Before 1989-90, ICD-8 codes were used; and from 1989-90 to 1997, ICD-9 codes were used. The change should not have influenced reporting of syphilis, gonorrhoea, trichomoniasis, or chancroid. There was no specific ICD-8 code for pelvic inflammatory disease. In any case, the changes implemented in 198990 would not have influenced trends recorded from 1989-90 onwards. For syphilis the codes used were 091.0 and 097.0, for gonorrhoea 098.0, genital herpes 054.1, pelvic inflammatory disease 614.0, trichomoniasis 131.01, and chancroid 099.0. Results of laboratory testing done to support these diagnoses were not available, but likely testing was infrequent, except for syphilis. 


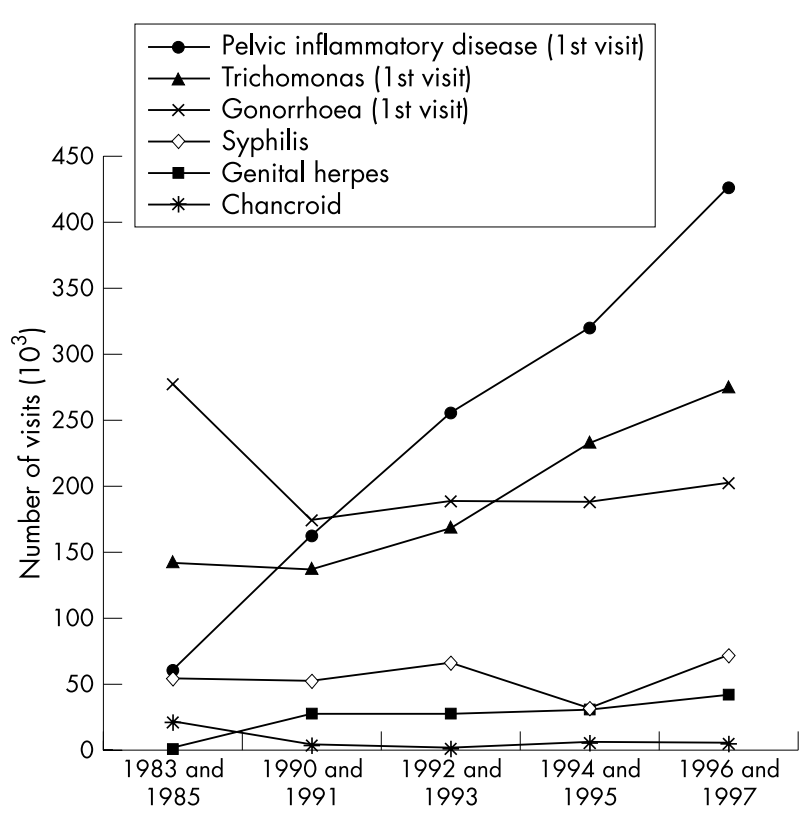

Figure 1 Trends in numbers of visits per 2 year period for different STD to physicians in private practice. Peru, 1983-97. Source: IMS Health.

\section{RESULTS}

First visits increased sevenfold over time for PID, and twofold for trichomoniasis (fig 1). Visits increased 2.6-fold for genital herpes. First visits for conditions coded as gonorrhoea showed a substantial decline from 276000 for $1983-5$ to 173000 in 1990-1; since then first visits for gonorrhoea have not changed greatly. Visits for conditions coded as syphilis have remained relatively stable over time, with an estimated 72800 visits for syphilis in 1996-7. Small numbers of diagnoses of chancroid made estimates for this disease imprecise.

Data regarding age and sex were available for gonorrhoea. The declining ratio of numbers of males to females who received a diagnosis of gonorrhoea (from 5.3 in 1983-5 to 1.47 in 1996-7) reflected both an increase in numbers of diagnoses in females and a decrease in diagnoses in males over this time (fig 2 ).

\section{Treatments used}

Treatments prescribed for gonorrhoea, PID, and trichomoniasis were analysed.

\section{Gonorrhoea}

All patients given a diagnosis of gonorrhoea received an antibiotic at the first visit (table 1). The average number of antibiotics prescribed per visit was 1.15 for 1983 and 1985 and in 1996 and 1997. Spectinomycin was the most commonly prescribed antibiotic from 1983 and 1985 (53.6\% of 276000 first visits) through 1994-5 (35.1\% of 188000 first visits), after which aminoglycosides (gentamicin, kanamycin) were more frequently used. Use of third generation cephalosporins increased somewhat from 1992-3 to 1996-7, while use of tetracyclines declined substantially, indicating inadequate treatment for potentially coexisting chlamydial infection. Use of penicillins also declined after 1990-1, appropriate for Peru, where $40(64.5 \%)$ of 62 gonorrhoea isolates collected during 1999 were $\beta$ lactamase positive or had chromosomally mediated penicillin resistance (K Holmes, W Whittington, unpublished data).

\section{Pelvic inflammatory disease}

Of all first visits for PID, the percentage of cases given an antibiotic increased from $63.3 \%$ of 58000 in $1983-5$ to $87 \%$ of 428000 in 1996-7 (table 2). Treatment with sulfamethoxazole-trimethoprim dropped off sharply after 1983-5; while use of a drug highly active against chlamydia (tetracycline) or anaerobes (nitroimidazole or clindamycin) increased from less than $10 \%$ in $1983-5$, to about $40 \%$ and $25 \%$, respectively, during 1996-7. However, surprisingly few patients received drugs recommended for gonorrhoea (for example, aminoglycosides, fluoroquinolones). For example, in 1996-7, 15.6\% of first visit patients received an aminoglycoside, and $6.0 \%$ received a fluoroquinolone; none received a cephalosporin, spectinomycin, or azithromycin.

\section{Trichomoniasis}

Use of oral nitroimidazoles (metronidazole, tinidazole, secnidazole, nimorazole) decreased from $94.6 \%$ of first visits in $1983-5$, and $95.6 \%$ in $1990-1$, to $54.3 \%$ in 1996-7 (table 3). Throughout this period, use of intravaginal preparations increased from $66.8 \%$ in $1983-5$ and $50.7 \%$ in $1990-1$, to $104.3 \%$ in 1996-7. Thus oral metronidazole treatment

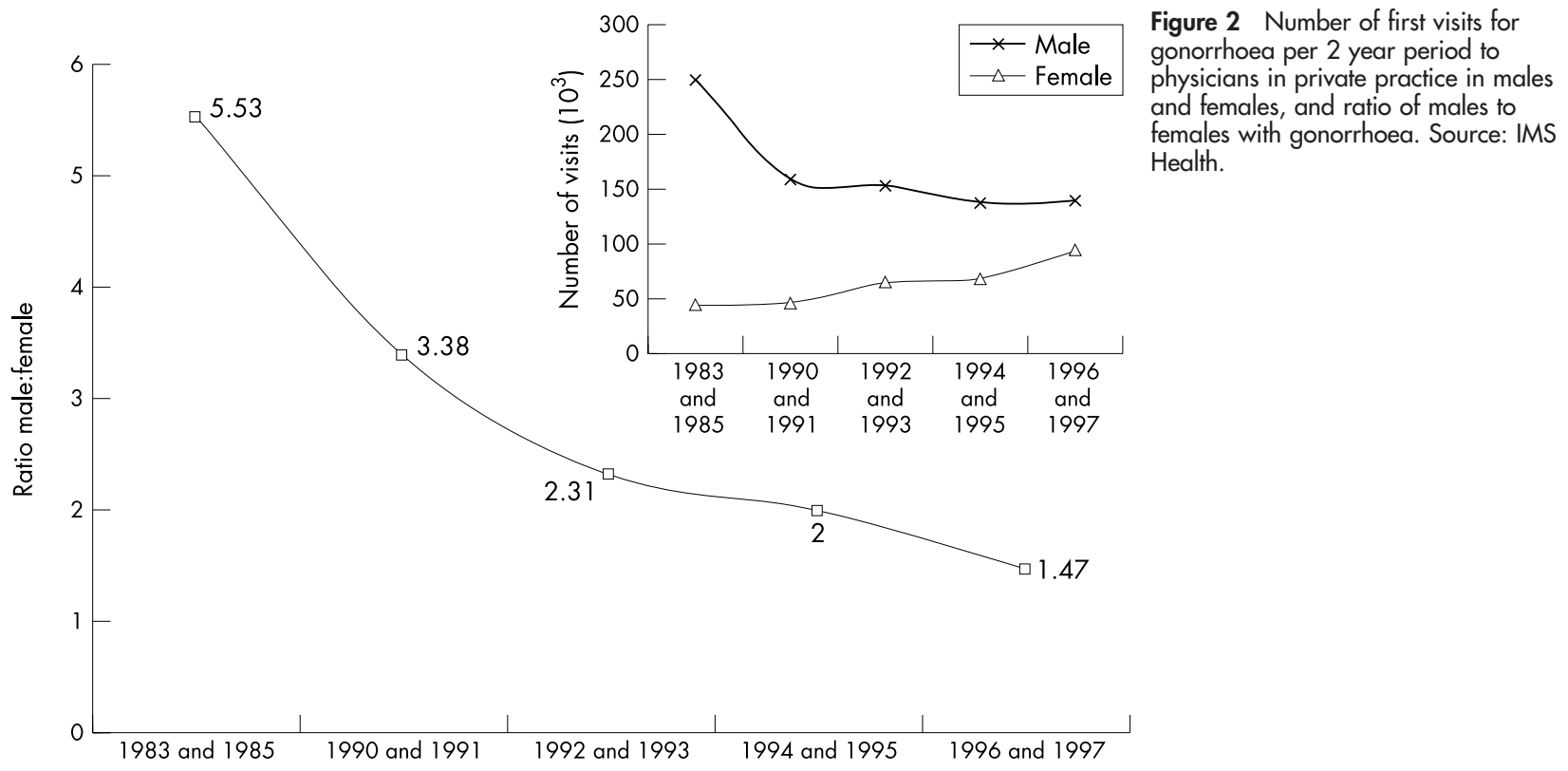


Table 1 Percentage distribution and estimated total number of antibiotics prescribed for the diagnosis of gonorrhoea by private physicians in Peru in the IMS sample, 1983-97

\begin{tabular}{llllll}
\hline Antibiotics & $\begin{array}{l}1983 \\
1985\end{array}$ & $\begin{array}{l}1990 \text { and } \\
1991\end{array}$ & $\begin{array}{l}1992 \text { and } \\
1993\end{array}$ & $\begin{array}{l}1994 \text { and } \\
1995\end{array}$ & $\begin{array}{l}1996 \text { and } \\
1997\end{array}$ \\
\hline Third generation cephalosporins & $0.0 \%$ & $5.3 \%$ & $1.4 \%$ & $18.3 \%$ & $20.1 \%$ \\
First generation cephalosporins & $0.0 \%$ & $2.7 \%$ & $0.0 \%$ & $3.4 \%$ & $4.2 \%$ \\
Fluoroquinolones & $0.0 \%$ & $3.1 \%$ & $5.0 \%$ & $0.0 \%$ & $9.6 \%$ \\
Spectinomycin & $53.5 \%$ & $61.9 \%$ & $50.0 \%$ & $34.5 \%$ & $24.6 \%$ \\
Tetracyclines & $10.5 \%$ & $12.0 \%$ & $18.4 \%$ & $16.4 \%$ & $5.4 \%$ \\
Penicillins & $14.6 \%$ & $16.6 \%$ & $13.9 \%$ & $7.8 \%$ & $10.6 \%$ \\
Aminoglycosides & $25.6 \%$ & $22.2 \%$ & $18.2 \%$ & $22.7 \%$ & $33.8 \%$ \\
Estimated total number of antibiotics & 307 & 220 & 231 & 209 & 226 \\
prescribed for gonorrhoea $\times 10^{3}$ & & & & &
\end{tabular}

actually became less frequent, while use of intravaginal preparations increased and were commonly used together with oral nitroimidazoles throughout this period. Of the intravaginal antimicrobial preparations used for trichomoniasis, the variety of combinations and types of preparations used is remarkable.

Frequently more than one drug of any type was given for vaginal trichomoniasis (estimated average of 1.61 drugs per visit prescribed at 184000 visits for trichomoniasis in 1983-5, and 1.59 drugs per visit prescribed at 311000 visits in 1996-7).

\section{DISCUSSION}

These data showed increasing numbers of patients making first visits to physicians in private practice for PID and trichomoniasis, and an increase in total visits for genital herpes over a 15 year period in Peru. The male to female ratio for gonorrhoea declined because of increasing numbers of cases diagnosed in women and decreasing numbers in men.

Surprisingly, treatment for gonorrhoea usually involved use of spectinomycin or an aminoglycoside, with a shift from the former to the latter. Use of first line treatments for gonorrhoea, ${ }^{11}{ }^{12}$ such as third generation cephalosporins or fluoroquinolones, increased but still remained uncommon. Physicians seldom prescribed a tetracycline for gonorrhoea, even though clinicians in Peru seldom differentiate gonorrhoea from NGU, or test for chlamydial infection in cases of urethritis. Treatment offered at first visits for PID seldom provided effective treatment for gonorrhoea, chlamydial infection, or anaerobic infection. For trichomoniasis, the declining use of oral nitroimidazoles was disconcerting; the most common treatments prescribed involved preparations not shown to be effective in published well controlled clinical trials; some lacked well established biological activity against Trichomonas vaginalis (or bacterial vaginosis, a common cause of vaginal discharge probably seldom differentiated from trichomoniasis in Peru). Polycresulen ovules can produce vaginal ulcerations. ${ }^{13}$
Although clinicians are required to report cases of STD (anonymously) in Peru, physicians in private practice rarely do so. No other data on trends on STD seen by private physicians or on treatments used are available in Peru or in most other Latin American countries.

With offices in more than 70 countries and information services in over 100 countries, IMS Health tracks patient consultations and treatments prescribed by private physicians in the world's major countries, where ongoing IMS surveys present an interesting surveillance opportunity. The number of cases of STD syndromes seen by physicians in private practice, as estimated from this active surveillance, can be compared with other estimates in Peru. For example, the Demographic and Health Survey (DHS) in Peru in 1996 posed a set of questions to representative samples of 32646 women 15-49 years old, and of 3201 men 15-59 years old, asking about history of urethral discharge, and diagnosis of gonorrhoea during the past year. The Ministry of Health $(\mathrm{MOH})$ of Peru also collects data on numbers of cases of selected STD syndromes seen in $\mathrm{MOH}$ clinics. The estimated 65000 cases of "gonorrhoea" in men of all ages seen by physicians in private practice in the IMS Health audit in 1996 can be compared with 63375 cases of urethral discharge or gonorrhoea in men estimated from the 1996 DHS survey, 8503 cases of urethral discharge in men seen in MOH clinics in 1997. In 1995-6, a survey of a random sample of pharmacies in Lima, pharmacy workers retrospectively reported having seen 398 client visits for urethral discharge in men per 100000 population of Lima per month; this can be compared with 159 first visits to physicians in private practice in 1996 for "gonorrhoea" in men per 100000 population of Lima estimated from the 1996 IMS Health survey. ${ }^{14}$

Thus, although the numbers of men surveyed in the 1996 DHS was probably too small to give precise estimates for urethritis in men in Peru, the other surveys suggest the largest number of cases of urethritis in men may be seen in

Table 2 Percentage distribution and estimated total number of antibiotics prescribed for the diagnosis of pelvic inflammatory disease by private physicians in Peru in the IMS sample

\begin{tabular}{llllll}
\hline Antibiotics & $\begin{array}{l}1983 \\
1985\end{array}$ & $\begin{array}{l}1990 \text { and } \\
1991\end{array}$ & $\begin{array}{l}1992 \text { and } \\
1993\end{array}$ & $\begin{array}{l}1994 \text { and } \\
1995\end{array}$ & $\begin{array}{l}1996 \text { and } \\
1997\end{array}$ \\
\hline Fluoroquinolones & $0.0 \%$ & $0.0 \%$ & $0.0 \%$ & $5.1 \%$ & $7.3 \%$ \\
Tetracyclines & $3.1 \%$ & $35.3 \%$ & $61.9 \%$ & $42.2 \%$ & $40.1 \%$ \\
Penicillins & $14.1 \%$ & $8.1 \%$ & $6.1 \%$ & $4.4 \%$ & $0.0 \%$ \\
Aminoglycosides & $0.0 \%$ & $1.1 \%$ & $4.7 \%$ & $9.5 \%$ & $5.5 \%$ \\
Nitroimidazole or clindamycin & $3.1 \%$ & $3.7 \%$ & $0.0 \%$ & $24.7 \%$ & $26.2 \%$ \\
Trimethoprim-sulfamethoxazole & $42.2 \%$ & $0.0 \%$ & $4.9 \%$ & $0.0 \%$ & $0.0 \%$ \\
Estimated total number of antibiotics & 36 & 86 & 211 & 316 & 372 \\
prescribed for PID $\times 10^{3}$ & & & & &
\end{tabular}


Table 3 Percentage distribution and total number of medications prescribed for the diagnosis of vaginal trichomoniasis by private physicians in Peru in the IMS sample

\begin{tabular}{|c|c|c|c|c|c|}
\hline & $\begin{array}{l}1983 \text { and } \\
1985\end{array}$ & $\begin{array}{l}1990 \text { and } \\
1991\end{array}$ & $\begin{array}{l}1992 \text { and } \\
1993\end{array}$ & $\begin{array}{l}1994 \text { and } \\
1995\end{array}$ & $\begin{array}{l}1996 \text { and } \\
1997\end{array}$ \\
\hline $\begin{array}{l}\text { Oral nitroimidazoles* } \\
\text { Intravaginal preparations } \dagger\end{array}$ & $94.6 \%$ & $95.6 \%$ & $61.8 \%$ & $65.8 \%$ & $54.3 \%$ \\
\hline Metronidazole & $0.0 \%$ & $3.1 \%$ & $12.0 \%$ & $9.0 \%$ & $2.6 \%$ \\
\hline Nitroimidazole + antifungal & $42.9 \%$ & $36.3 \%$ & $53.1 \%$ & $62.6 \%$ & $75.9 \%$ \\
\hline Antifungal & $10.3 \%$ & $5.0 \%$ & $7.9 \%$ & $7.6 \%$ & $11.3 \%$ \\
\hline $\begin{array}{l}\text { Other intravaginal preparations } \\
\text { Other }\end{array}$ & $13.6 \%$ & $6.3 \%$ & $3.7 \%$ & $7.2 \%$ & $14.5 \%$ \\
\hline Solcotricovac (lactobacilli -injectable IM) & $0.0 \%$ & $1.9 \%$ & $0.0 \%$ & $0.0 \%$ & $0.0 \%$ \\
\hline $\begin{array}{l}\text { Estimated total number of medications } \\
\text { prescribed for trichomoniasis } \times 10^{3}\end{array}$ & 348 & 274 & 394 & 490 & 567 \\
\hline \multicolumn{6}{|c|}{$\begin{array}{l}\text { *Nitroimidazoles available orally include tinidazole, metronidazole, secnidazole, nimorazole, and ornidazole in } \\
\text { decreasing order of frequency. } \\
\text { †Topical nitroimidazoles include metronidazole or tinidazole. Antifungals used topically include nystatin, } \\
\text { clotrimazole, miconazole, tioconazole, isoconazole, boric acid with or without dexamethasone or with nifuratel, } \\
\text { and amphotericin plus tetracycline. Other intravaginal preparations include estriol, povidone-iodine, nitrofurans, } \\
\text { and polycresulen ovules. }\end{array}$} \\
\hline
\end{tabular}

pharmacies, followed by physicians in private practice, with fewer cases seen in $\mathrm{MOH}$ clinics.

Data from this study must be interpreted cautiously. Although data for gonorrhoea, PID, and trichomoniasis were for first visits, data for herpes and syphilis concerned all visits and may have overestimated the number of patients seen with these conditions. Further, the ICD-9 codes used may not be highly sensitive or specific for the STD syndromes of interest. Certain diagnoses (for example, non-gonococcal urethritis (NGU)) were too uncommon in the IMS survey to warrant estimates of number of visits; this suggests a tendency of physicians in the survey to overdiagnose gonorrhoea and underdiagnose NGU in the absence of laboratory testing. Similarly, the diagnosis of trichomoniasis may include several types of vaginal infections, and the bases for differentiating syphilis, genital herpes, and chancroid are uncertain, as is the accuracy of the clinical diagnosis of PID. These limitations are intrinsic to surveillance of STD syndromes in many settings. It remains uncertain how trends in frequency of visits for various STD syndromes truly reflect changing incidence, or changing patterns of diagnosis, $v$ changing patterns of healthcare seeking behaviour. Increasing awareness of physicians or patients about PID and herpes from the early 1980s to the mid-late 1990s could partly account for increasing numbers of visits recorded for these diagnoses.

Because we lacked information on the total number of visits for any diagnosis to private physicians during the period reviewed, the increasing number of cases of certain STD diagnosis could reflect increasing numbers of patients in general going to private physicians in the survey over time. The number of people attending private physicians is subject to shifts in healthcare utilisation from public to private sources and vice versa, and may obfuscate trends in disease incidence. A previous analysis by investigators from the US Center for Disease Control of NDTI data indicates this did not account for increasing numbers of visits over time for genital warts in the United States. ${ }^{8}$ For practical purposes an increasing number of visits to private physicians for STD, whatever the reason, underscores the urgent need to train these professionals in STD syndromic management. Finally, an important limitation is that patients seeking care from physicians in private practice probably under-represent individuals of lower socioeconomic status, who tend to have higher rates of STDs; and also may under-represent certain high risk populations such as female sex workers, men who have sex with men and youths.
The strengths of these data collected by IMS Health include the systematic well described methodology, using standardised ICD coding (now ICD-10) and a stratified sample of physicians in private practice. The system potentially allows monitoring of frequency of visits to physicians over time, not only for reportable diseases, but also for non-reportable diseases. Such data complement any data collected from surveillance of people attending STD clinics, and other high risk populations who represent target sentinel surveillance populations for syphilis and HIV infection. Because reporting from private sources is usually less complete than that by public sources, active surveillance of clinical encounters in the private sector is especially important. For example, in the United States, it has been estimated from self reported data in a national survey that treatment was received in an STD clinic for only $10.3 \%$ of cases of gonorrhoea, $8.9 \%$ of syphilis, and $8.7 \%$ of chlamydial infections. ${ }^{15}$

In summary, it appears that antimicrobials being used for "gonorrhoea," probably including most cases of urethral discharge in men, provide inadequate treatment for chlamydial infection and often include drugs which are active but second line choices for gonorrhoea. Treatment for PID and trichomoniasis (probably representing all vaginal discharge syndromes) appears grossly inadequate. The data highlight the need for improving STD management by physicians in private practice in Peru.

\section{ACKNOWLEDGEMENTS}

The authors gratefully acknowledge the help of IMS Health in providing the data for these analyses; and in making several helpful suggestions in the interpretation of the data.

Patricia J Garcia and King K Holmes both participated with IMS Health in identifying data needed for these studies, and carried out the analyses and preparation of the manuscript.

Supported by the University of Washington STD Cooperative Research Center (NIAID AI-31448); the Fogarty International Center (T22TW00001); the University of Washington International AIDS Research Training Program (NIAID AI-0714P); and an unrestricted grant for infectious diseases research from the Bristol Myers Squibb Corporation. Data for these analyses were kindly provided by IMS Health, Peru.

\section{Authors' affiliations}

P J Garcia, K K Holmes, Center for AIDS and STD, University of Washington, Seattle, WA, USA

P J Garcia, School of Public Health, Universidad Peruana Cayetano Heredia, Lima, Peru

Conflict of interest: None. 


\section{REFERENCES}

1 Aral SO, Holmes KK. Social and behavioral determinants of the epidemiology of STDs: industrialized and developing countries. In: Holmes KK, Mardh P-A, Sparling PF, et al, eds. Sexually transmitted diseases, 3rd ed. New York: McGraw-Hill, 1999:39-76.

2 Mindel A, Dalabetta G, Gerbase A, et al. Syndromic approach to STD management. Sex Transm Infect 1998;74(Suppl 1):S1-178.

3 Wawer MJ, Gray RH, Sewankambo NK, et al. Control of sexually transmitted diseases for AIDS prevention in Uganda: a randomized community trial. Rakai Project Study Group. Lancet 1999;353:525-35; and 1998;12:1211-25.

4 Pan American Health Organization. Informe anual de vigilancia del SIDA/ VIH/ETS. 1992. Washington DC, 1992.

5 Global Programme on AIDS. Global prevalence and incidence of selected curable sexually transmitted infections: overview and estimates, WHO/CDS/ CSR/EDC/2001.10. Washington DC: World Health Organization, 2001.

6 Gerbase A, Toscano C, Titan S, et al. Sexually transmitted diseases in Latin America and the Caribbean. Pan Am J Public Health 1999;6:362-70.

7 Division of STD Prevention. Sexually transmitted disease surveillance, 1998. Department of Health and Human Services. Atlanta: Centers for Disease Control and Prevention (CDC), September 1999.
8 Becker TM, Blount JH, Douglas J, et al. Trends in molluscum contagiosum in the United States, 1966-1983. Sex Transm Dis 1986;13:88-92.

9 Becker TM, Stone KM, Alexander ER. Genital human papillomavirus infection, a growing concern. Obstet Gynecol Clin North Am 1987;14:389-96.

10 Grimes DA, Blount JH, Patrick J, et al. Antibiotic treatment of pelvic inflammatory disease. Trends among private physicians in the United States, 1966 through 1983. JAMA 1986;256:3223-6.

11 Centers for Disease Control and Prevention. 1998 Guidelines for treatment of sexually transmitted diseases. Morb Mort Week Rep 1998;47(RR1): $1-116$.

12 World Health Organization. Management of patients with sexually transmitted diseases, GPA/TEM/94.1. Geneva: WHO, 1994.

13 Kilmarx P, Limpakarnjanarat K, Supawitkul S, et al. Mucosal disruption due to use of a widely-distributed commercial vaginal product: potential to facilitate HIV transmission. AIDS 1998;12:767-73.

14 Garcia PJ, Gotuzzo E, Hughes JP, et al. Syndromic management of STDs in pharmacies: evaluation and randomized intervention trial. Sex Transm Infect 1998;74(Suppl 1):S153-8.

15 Brackbill R, Sternberg M, Fishbein M. Where do people go for treatment of sexually transmitted diseases? Fam Plann Perspect 1999;31:10-15.

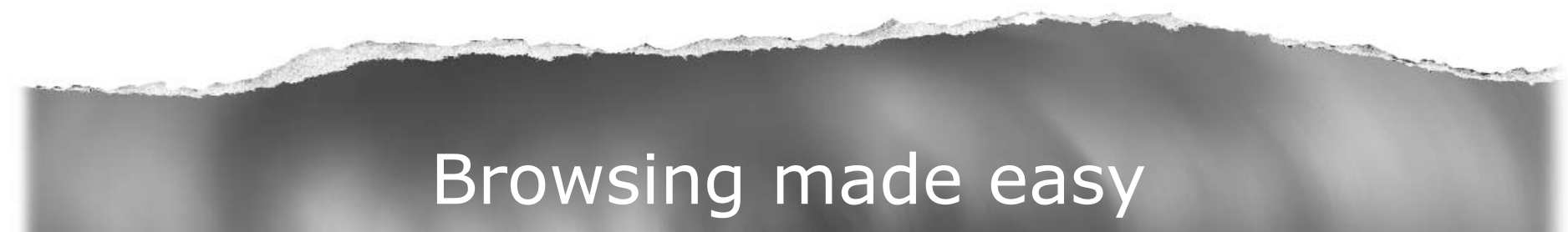

\section{Collections}

With a single click Collections allows you to find all articles that have been published in your chosen subject. Select from over 200 clinical and non-clinical topic collections and/or cross search other specialist journals, the BMJ and Cochrane Reviews

\section{www.sextransinf.com}

